\title{
Ein Kiesel fern der Brandung
}

Alois Gratwohl

Prof. Dr. med., emeritierter Ordinarius für Hämatologie und Stammzelltransplantation, Basel
Der Präsident der Nationalen Ethikkommission stellt in seinem Beitrag in der Schweizerischen Ärztezeitung Gedanken und Konzepte zur Ethik vor. Das ist gut so. Seine Darstellung erlaubt eine offene Diskussion über seine Sicht der Dinge. Sie führt konsequenterweise zur Frage, ob eine solche Ansicht an der Spitze der Institution NEK ihren Platz hat, in einem forschungsorientierten Land mit einer modernen, fortschrittlichen Medizin. Dieser Einwand richtet sich nicht gegen die NEK. Die Schweiz benötigt eine einem moralischen Mantel». Diese Unterstellung an sich ist inakzeptabel. In seiner abwertenden Haltung übersieht er, dass er mögliche allgemeingültige Prinzipien einer kantschen Fundamentalethik auf die zweite, die Sachebene, überträgt und übersieht Veränderungen der Zeit. Er verharrt im Denken, dass individuelles Leben absolut mit der befruchteten Eizelle beginnt. Dem ist nicht mehr so. Die Stammzellforschung hat gezeigt, dass somatische Zellen durch externe Faktoren Totipotenz wiedererlangen können.

\section{Neue wissenschaftliche Erkenntnisse verlangen immer ein Umdenken und neue Ansätze - das fällt schwer}

führungsstarke und kompetente nationale Ethikkommission. Im Zusammenhang mit dem neuen Gesetz über die Forschung am Menschen stehen dringende Fragen an. Koordination der kantonalen Ethikkommissionen, Abwägen im Dilemma zwischen Privatisierung des Gewinns und Sozialisierung der Kosten in Forschung und Medizin, Brückenbildung zwischen Rechten und Pflichten von Forschern und Probanden in der klinischen Forschung sind nur einige der offenen Themen. Keines schneidet Professor Höffe an. Mit seinem Gedankengut bietet er keine Hilfe zur Problemlösung in der Medizinethik. Es gibt nicht «gute» und «schlechte» Ethik. Wer mit Begriffen wie «unerschütterliche Grundsätze», «Atem von Jahrhunderten» und «kompromisslosen Vorrang der zwangsbefugten Elementarmoral» denkt, ist im newtonschen Denken verblieben und verkennt die Gesetze der Relativitätsund Quantentheorie. Nur im Museum ist der «UrMeter» eine absolute Grösse; je strikter und absoluter eine Definition, desto grösser die Unschärfe.

Ich gehe nicht auf den an sich spannenden Disput ein, ob eine kantsche oder utilitaristische Sichtweise für die Ethik «richtig» ist. Darüber dürfen Ethiker streiten. Ich bin nur äusserst unangenehm berührt, wenn der Leiter einer NEK einen Protagonisten der Utilitarier, Peter Singer, im Disput verunglimpft und dessen persönliche Familiengeschichte zur Gegenbeweisführung beizieht. Wer ist schon ohne Schuld? Ich konzentriere mich auf die offenen und versteckten Seitenhiebe gegen die Stammzellforschung.

Professor Höffe unterstellt Wissenschaftlern in der Stammzellforschung eine «Windfahnen-Ethik mit
Durch diese tierexperimentellen Befunde hat der Beginn individuellen Lebens eine neue Dimension erhalten. Eine reprogrammierte Fibroblasten- oder Hautzelle kann gleiches Potential wie eine befruchtete Eizelle besitzen. Diese Zellkultur ist dennoch kein Individuum mit besonderem Schutzanspruch. Sie verdient Umgang mit Fachwissen, Respekt und Kontrolle.

Mit der Reprogrammierung von somatischen Zellen ist Neuland betreten, das es vorher nicht gab. Es ist vergleichbar mit der ersten Herztransplantation, als der Herztod seine Berechtigung als wichtigster Messpunkt des individuellen Todes, das Herz seinen

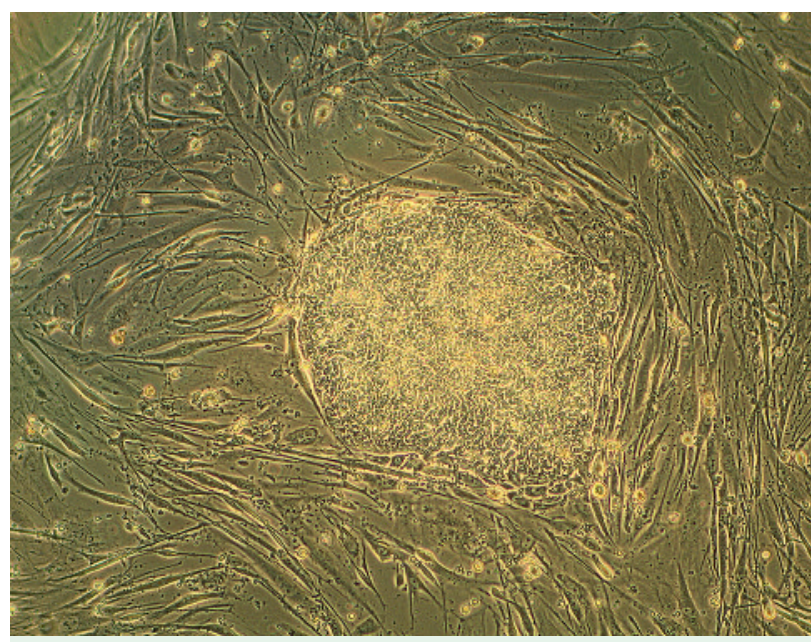

Kolonie embryonaler Stammzellen - neue Erkenntnisse der Forschung können ein Umdenken erfordern. 
Anspruch auf Sitz der Seele verlor. Gesetzgeber, Ethik und Wissenschaft haben die Herausforderung angenommen, den Zeitpunkt des Todes neu zu definieren. Nicht Wetterfahnen führten zu diesem Prozess, sondern neue wissenschaftliche Befunde. Das Resultat ist bemerkenswert. Nicht der Zeitpunkt des Todes wird gemessen; definierte Kriterien erlauben festzustellen, dass der Tod eingetreten ist. Der «Unschärfe» wird Rechnung getragen. Vergleichbar sind heute Gesellschaft, Gesetzgeber, Ethik und Wissenschaft gefordert, sich mit dem Beginn individuellen Lebens auseinanderzusetzen. Es ist vorauszusehen, dass die Fixierung auf «einen Moment» nicht die Lösung sein wird. Neue Begriffe sind notwendig; «Embryo» wird als Bezeichnung ein historisches Relikt. Für diese dringende Aufgabe der Begriffsfindung und Nomenklatur sind alle gefordert, auch die Ethik. Mit der absolutistischen Denkweise von Professor Höffe kann diese
Hilfe nicht geboten werden. Sie ist rückwärtsorientiert. Schon Moses war weise; er brachte 10 Gebote, nicht 10 Verbote Gottes.

Die zugrunde liegende Problematik ist dabei alt und wiederholt sich immer wieder. Neue wissenschaftliche Erkenntnisse verlangen immer ein Umdenken und neue Ansätze. Das fällt schwer. Die Abkehr vom geozentrischen Weltbild ist ein bekanntes Beispiel. Weder Verbote, Bannstrahl oder Argumente von «Jahrhunderte oder Jahrtausende an vorherige Festhalten» brachten die Erde in den Mittelpunkt des Sonnensystems zurück. Professor Höffe wird meinen Argumenten die Prinzipienstärke, den Fels in der Brandung erst recht entgegenhalten. Nur, auch Gestade ändern sich. Der Fels in der Brandung wird zum bedeutungslosen Kiesel fern der Brandung. Es scheint dringend, die Aufgaben der NEK und deren Leitung zu diskutieren. 